Review

\title{
Management of acute cardiovascular events in patients with COVID-19
}

\author{
Chia-Te Liao ${ }^{1,2,3}$, Wei-Ting Chang ${ }^{1,4,5}$, Wen-Liang Yu ${ }^{6,7}$ and Han Siong Toh ${ }^{4,6, *}$ \\ ${ }^{1}$ Department of Cardiology, Chi Mei Medical Center, 71004, Tainan, Taiwan \\ ${ }^{2}$ Department of Public Health, College of Medicine, National Cheng Kung University, 70101, Tainan, Taiwan \\ ${ }^{3}$ Department of Electrical Engineer, Southern Taiwan University of Science and Technology, 71005, Tainan, Taiwan \\ ${ }^{4}$ Institute of Clinical Medicine, College of Medicine, National Cheng Kung University, 70101, Tainan, Taiwan \\ ${ }^{5}$ Department of Biotechnology, Southern Taiwan University of Science and Technology, 71005, Tainan, Taiwan \\ ${ }^{6}$ Department of Intensive Care Medicine, Chi Mei Medical Center, 71004, Tainan, Taiwan \\ ${ }^{7}$ Department of Medicine, School of Medicine, College of Medicine, Taipei Medical University, 1 10, Taipei, Taiwan \\ *Correspondence: kampungths@gmail.com (Han Siong Toh)
}

DOI: $10.31083 /$ i.rcm.2020.04.140

This is an open access article under the CC BY 4.0 license (https://creativecommons.org/licenses/by/4.0/).

The pandemic of coronavirus disease 2019 (COVID19) caused by the newly discovered virus, severe acute respiratory syndrome coronavirus 2 (SARS-CoV-2), had been noticed to have high morbidity and mortality. Apart from pneumonia, COVID-19 can also cause damage to the cardiovascular system, and co-occurring with cardiovascular injury leads to a poorer prognosis. Besides, amid the pandemic of COVID-19, the management of critical cardiovascular events needs to further account for the highly infectious coronavirus, prompt and optimal treatments, clinician's safety, and healthcare provider's capacity. This review article aims to provide more comprehensive and appropriate guidance for the management of critical cardiovascular disease, including ST-segment elevation myocardial infarction (STEMI), non-STEMI acute coronary syndrome, cardiogenic shock, acute heart failure, cardiopulmonary resuscitation, and advanced care planning, during the COVID-19 epidemic.

\section{Keywords}

COVID-19; critical cardiovascular diseases; resuscitation; advanced care planning

\section{Background}

The coronavirus disease 2019 (COVID-19) caused by severe acute respiratory syndrome coronavirus 2 (SARS-CoV-2) has had an enormous impact on public health (The World Health Organization, 2020). This communicable disease does not only damage the respiratory organs but also influences other systems (Dong et al., 2020; Guo et al., 2020; Inciardi et al., 2020; Shi et al., 2020; Zheng et al., 2020). Evidence has indicated that COVID-19 leads to myocardial injury and the incidence is up to $10-20 \%$ (Clerkin et al., 2020; Shi et al., 2020). The exact mechanism has not been elucidated, but plausible explanations may be the direct damage from SARS-CoV-2, plaque rupture, hypoxia injury, sequent cytokine storm, coronary spasm, microthrombi, and overacting of immune systems (Pedersen and Ho, 2020; Tavazzi et al., 2020). COVID-19 patients who exhibit myocardial injury may have elevated troponin-I levels, and the magnitude is associated with patients' prognoses (Zheng et al., 2020; Zhou et al., 2020). Apart from myocardial injury, the patients have poorer prognoses, more critical illnesses, and higher mortalities given the co-occurrence of COVID-19 and underlying cardiovascular diseases (Bonow et al., 2020; Clerkin et al., 2020; He et al., 2020; Shi et al., 2020).

Although some countries have flattened the curve of COVID19, the global COVID-19 pandemic remains wreaking havoc on the healthcare system. Particular, the patients living with cardiovascular diseases have poorer prognosis when suffering SARS-CoV-2 infection. Amid the era of COVID-19, the management of critical cardiovascular diseases does not only emphasize efficacious and prompt cares, but also need to account for the appropriateness of treatment strategies and safety of medical staff. As a result, this paper aimed to review the current studies and provide comprehensive managements regarding critical cardiovascular diseases during COVID-19.

\section{Management of acute coronary syndrome in patients with COVID-19 \\ 2.1 Acute coronary syndrome}

Acute coronary syndrome (ACS) is a syndrome describing myocardial ischemia/injury due to decreased blood flow in the coronary artery. ACS comprises ST-segment elevation myocardial infarction (STEMI) and non-ST elevation ACS, which includes nonST segment elevation myocardial infarction (NSTEMI) and unstable angina. Moreover, there are two major types of myocardial infarction (Thygesen et al., 2018). Type 1 myocardial infarction is an atherothrombotic coronary artery disease, and blood flow is 
usually obstructed by the rupture or erosion of the atherosclerotic plaque. Type 2 myocardial infarction is due to a mismatch between oxygen supply and demand. Among COVID-19 patients, type 2 myocardial infarction makes up the majority, and these patients are likely to have a lower Troponin-I peak, but a higher risk of intubation, shock, and mortality in hospital, compared to those with real obstructive coronary artery disease (Bangalore et al., 2020).

After the outbreak of COVID-19, the pandemic has massively changed the daily practice of the catheterization laboratory (cath lab). Particularly, how to give prompt coronary artery reperfusion and protect the frontline medical staff from COVID-19 infection has become a challenge. Currently, many institutes and scientific societies have already provided their recommendations and experiences (Campo et al., 2020; Jing et al., 2020; Murthy et al., 2020; Welt et al., 2020). From the experience in Italy, the safety of the cath-lab team is the first priority because medical volume needs to be preserved to save more patients (Campo et al., 2020). Also, sufficient preparation of personal protective equipment (PPE), training programs, and establishing control centers in advance are crucial and necessary (Campo et al., 2020). Although there has been no consensus concerning the construction of a negative-pressure catheterization room, daily checks of adequate PPE, familiarity with donning and doffing PPE and standard workflow, help prevent the staff from SARS-CoV-2 infection. If the institute is not overwhelmed by the pandemic, the cath lab may set up a designated room for confirmed or suspected COVID-19 patients (Welt et al., 2020). Meanwhile, the equipment of percutaneous coronary intervention (PCI) should be placed in a closed cabin of the room before the patient arrives. After the procedure, sterilization of the uncovered equipment in the cabin is thought to be unnecessary, but the equipment exposed to the patient's aerosolization or droplets during the procedure, i.e., intubation, and cardiopulmonary resuscitation, should be sterilized prudently or discarded directly. The cath lab may request minimal or designated staff to enter the room and avoid repeated entry of both patients and staff. Besides, multidisciplinary collaboration between various departments, including cardiology, emergency, infections, pneumology, radiology, and the medical laboratory department, is required for things to go smoothly (Jing et al., 2020). Until now, there has been a lack of recommendations on the management of COVID-19 patients when blood is exposed. The disposal of sterilized drapes and equipment may be useful, and medical staff at risk of bloodstream infection may follow the original management procedures.

\subsection{ST-segment elevation myocardial infarction (STEMI)}

Although SARS-CoV-2 infection leads to an increase in myocardial injury, several national statistics have revealed a notable drop in STEMI catheterization activation amid the COVID-19 pandemic (a 40\% drop in Spain, and a 38\% drop in the U.S.) (Garcia et al., 2020; Rodríguez-Leor et al., 2020). There are several plausible explanations for this phenomenon including avoidance of hospital visits due to fear of getting COVID-19, STEMI misdiagnosis, increased medical reperfusion, reduced daily stressors due to social distancing and quarantine, less pollution from traffic and factories, more rest and less activity, and less smoking due to warnings from social media (Garcia et al., 2020; Rodríguez-Leor et al., 2020).

Amid the COVID-19 pandemic, clinicians may need to take into account the local healthcare provider's regulations, medical resources, the volume of PPE and the intensity of the pandemic for the management of STEMI. Besides, medical staff should prudently differentiate between myocarditis, myocardial injury, and STEMI for COVID-19 patients. All STEMI patients should be tested for SARS-Cov-2 as soon as possible after their first medical contact. Before the SARS-Cov-2 test results in STEMI patients are available, any STEMI patient should be treated as potentially infected, and all protective measures should be taken to avoid potential infection of medical staff and other patients.

Coronary reperfusion therapy in ACS patients with ischemia symptoms or persistent ST elevation in the two least continuous electrocardiogram leads is still indicated within 12 hours (Ibanez et al., 2018). The goal of timely reperfusion remains the same, but the safety of healthcare providers should be ensured. Contrary to the evidence, some healthcare providers switched to a fibrinolysisbased strategy for STEMI patients, even though the therapy has been proven to be inferior in clinical trials (Jing et al., 2020). The various approaches and protocols have left cath labs debating over whether to continue providing primary PCI; and this dilemma is dependent upon the intensity of the COVID-19 pandemic. If institutes are not overwhelmed by the pandemic and are able to provide a safe environment for medical staff and other patients, primary PCI remains the best option for reperfusion therapy. STEMI patients who tested negative for SARS-CoV-2 should be managed using the standard approach of primary PCI but also wear a mask. Nevertheless, under the current circumstances, primary PCI may be delayed due to the waiting time of SARS-CoV-2 test results and the implementation of protective measures. The European Society of Cardiology (ESC) recommended that the maximum delay from STEMI diagnosis to reperfusion in the COVID-19 era should not exceed 120 minutes (extra delayed time can reach up to 60 minutes from shared experiences) (Roffi et al., 2020; The European Society for Cardiology , 2020). For overwhelmed healthcare providers, fibrinolysis should be considered as first-line therapy if they cannot meet the recommended timeline and if the patient does not have a contraindication of fibrinolysis (Daniels et al., 2020; Roffi et al., 2020). All physicians involved in the STEMI protocol should be familiar with the administration of fibrinolysis, as well as the indications and contraindications in different scenarios. When performing PCI for ACS patients, left ventriculography is suggested to reduce the need for echocardiography and shorten the hospital stay. To avoid staged PCI and reduce hospital stay, interventional cardiologists may consider completing revascularization immediately given the proper situation. For non-culprit lesions, PCI may be postponed until the next admission after the peak of the outbreak. Nevertheless, PCI may be arranged during the same hospitalization if ischemic symptoms and hemodynamic instability remain, or if the lesion is angiographically unstable.

\subsection{Non-STEMI acute coronary syndrome}

Non-STEMI ACS is an urgent condition, but emergent PCI is usually not necessary. During the pandemic, physicians need to cautiously evaluate the patient for type 1 myocardial infarction because type 2 myocardial infarction, myocarditis, or myocardial injury resulting from respiratory failure or stress-induced cardiomyopathy are prevalent among COVID-19 patients (Li et al., 2020). Non-invasive diagnostic images, like coronary computed tomography angiography and echocardiography, may help the differential 
diagnosis. SARS-CoV-2 tests are suggested for all non-STEMI ACS patients as soon as possible after the first medical contact. Treatment strategies vary according to risk stratifications (Roffi et al., 2016). The ESC divided non-STEMI ACS patients into four groups, including very high risk, high risk, intermediate-risk, and low risk (The European Society for Cardiology , 2020). Very high risk comprises the following conditions: cardiogenic shock or hemodynamic instability, repetitive angina, fatal arrhythmia or cardiac arrest, mechanical complications of myocardial infarction, acute heart failure, and recurrent ST-segment elevation. It is suggested that management for this group follow the STEMI pathway. High-risk patients who display a rise in troponin levels and dynamic ECG changes or refractory ischemic symptoms should be treated with early intervention after the result of the SARS-CoV-2 test. The target timeframe should be within 24 hours of coronary revascularization. If the patients are confirmed COVID-19 cases, they may be sent to other equipped and capable hospitals. If the patients have intermediate risk, i.e., a rise in troponin accompanied with diabetes, renal insufficiency, reduced ejection fraction, or prior myocardial infarction, or have a low risk, non-invasive strategies, like medical treatment, and coronary computed tomography angiography for differential diagnosis are suggested. Early discharge with regular clinical follow-up visits would be arranged for these patients who only require non-invasive treatment and this would help avoid infection and utilization of additional healthcare resources.

\subsection{Cardiogenic shock}

Cardiogenic shock occurring in the COVID-19 patients may result from direct cardiogenic causes or concomitant myocardial suppression by hypoxia, systemic hyper inflammation response, and viremia vasodilation. The latter is more difficult to manage because of the complicated etiology, and multidisciplinary care may provide better quality healthcare. During the COVID-19 pandemic, several factors need to be considered for the management of cardiogenic shock. First, in general, management can follow current evidence-based guidelines (Thiele et al., 2019). Although healthcare providers aim to save as many lives as possible, allocation of resources requires a team-based consensus, particularly regarding the indication and contraindication for mechanical circulatory support, given the scarcity of resources during COVID-19. The management should take into account clinical benefits, patient's preference for end of life, equity, local culture, the healthcare system, and the resources of healthcare providers. As a result, in case there is a need for mechanical circulatory support, e.g., extracorporeal membrane oxygenation (ECMO), temporary ECMO is preferred for patients with oxygenation deficiency. Second, measures to protect the medical staff from SARS-CoV-2 infection are crucial when managing cardiogenic shock. Any cardiogenic shock patient should be tested for SARS-CoV-2 and should be treated as confirmed COVID-19 patients before the results are obtained. In the case of fever and signs of clinical infection, repeated tests are also required for patients. Also, invasive coronary angiography is usually needed for patients with cardiogenic shock, and corresponding protection measures for COVID-19 should be strictly employed by the cath lab.

\subsection{Acute heart failure}

In severe COVID-19 patients, concomitant acute heart failure is prevalent, which may be caused by myocardial injury, inflammation, or infarction, stress-induced cardiomyopathy, respiratory distress, multiorgan failure, and fluid overload or dehydration (Ruan et al., 2020). For hospitalized patients who develop heart failure, the mortality rate becomes higher, compared to those without heart failure (Zhou et al., 2020). Clinical presentations and non-invasive image findings, like batwing pattern, Kerley B line, bilateral pleural effusion, and cardiomegaly on chest X-ray or reduced ejection fraction on echocardiography, are crucial diagnostic tools. Medical staff should be familiar with donning and doffing the PPE and have available protection measures in place to avoid contaminant infection when performing echocardiography or other examinations. In addition, except for the elevated troponin from cardiac damage, a significant rise in BNP/NT-pro BNP can also help detect acute heart failure, and the biomarkers are likely to be associated with the prognosis (Shi et al., 2020). Until now, data on the treatment of acute heart failure in COVID-19 patients are still sparse. It is suggested that treatment strategies follow the medical therapy guidelines irrespective of whether the patients test positive or negative for COVID-19 (The European Society for Cardiology , 2020).

\subsection{Cardiopulmonary resuscitation}

The administration of cardiopulmonary resuscitation (CPR) in COVID-19 patients follows the guideline of advanced cardiac life support (ACLS) (Panchal et al., 2019). Nevertheless, during the outbreak of a pandemic, we need to ensure both prompt and optimal resuscitation for patients and safety for rescuers. Despite the utilization of PPE, healthcare professionals of resuscitation remain at a high risk of exposure to SARS-CoV-2 infection. Aerosol viral particles may be produced by chest compression, intubation, and positive pressure ventilation during CPR. Also, rescuers come into close contact with COVID-19 patients, and emergent events may lead to the omission of protection measures. Therefore, the American Heart Association provided updated recommendations for such situations, including reducing exposure to COVID-19, reducing risk of aerosolization by prioritizing oxygenation and ventilation strategies, and evaluating the appropriateness of resuscitation (Edelson et al., 2020). In terms of reducing exposure to COVID-19, all rescuers should be familiar with donning and doffing PPE, and PPE should be worn when administering resuscitation. There should be designated CPR providers, or the number of providers in the room should be limited. Mechanical CPR devices can replace manual chest compression, and all rescuers should be clear on the status of the patient when handing over to the next staff. A high efficiency particulate air (HEPA) filter should be attached for any manual or mechanical ventilation to avoid aerosol spreading, and patients in cardiac arrest should be intubated with a cuffed tube. Before intubation, bag-valve-mask ventilation connected with a HEPA filter and tight seal should be used to help oxygenation. Professionals most capable of first-pass intubation should be designated, and chest compression should be paused when intubating. Video laryngoscopy may help reduce exposure to aerosols, and manual ventilation with supraglottic airway or laryngeal mask may be considered for delayed intubation. Disconnection of the closed-circuit should be avoided by health- 
care providers. Last, the appropriateness of starting and terminating CPR should be evaluated by healthcare providers according to the likelihood of survival, the patient's condition, the capacity of providers, and regional medical resources.

\subsection{Advanced care planning}

Healthcare systems now face a difficult situation due to the outbreak of COVID-19. COVID-19 patients who are older and have more comorbidities or chronic illnesses may have poorer outcomes and are at a higher risk of death. Moreover, survivors undergoing acute respiratory distress syndrome may have limited respiratory function and lower quality of life. High-intensive care and nonbeneficial treatment to sustain life should be avoided, given that the patients agree. Also, high-intensive treatment may increase the spread of SARS-CoV-2 between patients, clinicians, and family members; this treatment also undoubtedly puts strains on healthcare capacity. Therefore, advanced care planning should be prioritized and should be discussed before acute critical illness occurs. Patients' goals and expectations should be assessed carefully, and discussions with patients or their proxy should be concordant with the patients' wishes for the administration of CPR and ACLS. Clinicians should avoid non-beneficial CPR. On the other hand, a donot-resuscitate order for appropriate patients during the COVID-19 pandemic should be determined by healthcare providers.

\section{Conclusions}

SARS-CoV-2 infection may lead to new cardiac injuries and worsen underlying cardiovascular diseases. Simultaneously, COVID-19 patients presenting a pattern of cardiovascular emergent events and damage consequently have poorer prognoses. The highly infectious coronavirus, prompt and optimal treatments, clinician's safety, healthcare provider's capacity should be taken into account when considering the appropriate management of critical cardiovascular diseases Health policies and procedures should be flexibly adjusted to regional conditions.

\section{Author contributions}

CTL had a contribution to the conception or design of the work and drafting of the manuscript. WLY contributed to the interpretation of data for this work. WTC and HST critically revised the manuscript. All authors gave final approval and agreed to be accountable for all aspects of work ensuring integrity and accuracy.

\section{Ethical approval and consent to participate}

All procedures performed in studies involving human participants were in accordance with the ethical standards of the institutional and/or national research committee and with the 1964 Helsinki declaration and its later amendments or comparable ethical standards. This is a review article and there are no participants in the study.

\section{Acknowledgements}

We would like to thank Dr. Hung-Jen Tang, the chief of Infection Control Center of Chi Mei Medical Center, for the advisement on infection control measures.

\section{Conflict of Interest}

All authors are free of conflict of interest Funding.

\section{Consent for publication}

All authors have read and approved the publication of final manuscript.

\section{Availability of supporting data}

This is a review article, and all supporting data came from currently existing studies.

Submitted: July 17, 2020

Revised: November 06, 2020

Accepted: November 08, 2020

Published: December 30, 2020

\section{References}

Bangalore, S., Sharma, A., Slotwiner, A., Yatskar, L., Harari, R., Shah, B., Ibrahim, H., Friedman, G. H., Thompson, C., Alviar, C. L., Chadow, H. L., Fishman, G. I., Reynolds, H. R., Keller, N. and Hochman, J. S. (2020) ST-segment elevation in patients with COVID-19 - a case series. New England Journal of Medicine 382, 2478-2480.

Bonow, R. O., Fonarow, G. C., O'Gara, P. T. and Yancy, C. W. (2020) Association of coronavirus disease 2019 (COVID-19) with myocardial injury and mortality. JAMA Cardiology 5, 751-753.

Campo, G., Rapezzi, C., Tavazzi, L. and Ferrari, R. (2020) Priorities for Cath labs in the COVID-19 tsunami. European Heart Journal 41, 1784-1785.

Clerkin, K. J., Fried, J. A., Raikhelkar, J., Sayer, G., Griffin, J. M., Masoumi, A., Jain, S. S., Burkhoff, D., Kumaraiah, D., Rabbani, L., Schwartz, A. and Uriel, N. (2020) COVID-19 and Cardiovascular disease. Circulation 141, 1648-1655.

Daniels, M. J., Cohen, M. G., Bavry, A. A. and Kumbhani, D. J. (2020) Reperfusion of STEMI in the COVID-19 Era-Business as usual? Circulation 141, 1948-1950.

Dong, N., Cai, J., Zhou, Y., Liu, J. and Li, F. (2020) End-stage heart failure with COVID-19. JACC: Heart Failure 8, 515-517.

Edelson, D. P., Sasson, C., Chan, P. S., Atkins, D. L., Aziz, K., Becker, L. B., Berg, R. A., Bradley, S. M., Brooks, S. C., Cheng, A., et al. (2020) Interim guidance for basic and advanced life support in adults, children, and neonates with suspected or confirmed COVID-19: from the emergency cardiovascular care committee and get with the guidelines $($ resuscitation adult and pediatric task forces of the American Heart Association. Circulation 141, e933-e943.

Garcia, S., Albaghdadi, M. S., Meraj, P. M., Schmidt, C., Garberich, R., Jaffer, F. A., Dixon, S., Rade, J. J., Tannenbaum, M., Chambers, J., Huang, P. P. and Henry, T. D. (2020) Reduction in ST-Segment elevation cardiac catheterization laboratory activations in the United States during COVID-19 pandemic. Journal of the American College of Cardiology 75, 2871-2872.

Guo, T., Fan, Y., Chen, M., Wu, X., Zhang, L., He, T., Wang, H., Wan, J., Wang, X. and Lu, Z. (2020) Cardiovascular implications of fatal outcomes of patients with coronavirus disease 2019 (COVID-19). JAMA Cardiology 5, 811-818.

He, X. W., Lai, J. S., Cheng, J., Wang, M. W., Liu, Y. J., Xiao, Z. C., Xu, C., Li, S. S. and Zeng, H. S. (2020) Impact of complicated myocardial injury on the clinical outcome of severe or critically ill COVID-19 patients. Zhonghua Xin Xue Guan Bing Za Zhi 48, 456-460.

Ibanez, B., James, S., Agewall, S., Antunes, M. J., Bucciarelli-Ducci, C., Bueno, H., Caforio, A. L., Crea, F., Goudevenos, J. A. and Halvorsen, S. (2018) 2017 ESC guidelines for the management of acute myocardial infarction in patients presenting with ST-segment elevation: The Task Force for the management of acute myocardial infarction in patients presenting with ST-segment elevation of the European Society of Cardiology (ESC). European Heart Journal 39, 119-177.

Inciardi, R. M., Lupi, L., Zaccone, G., Italia, L., Raffo, M., Tomasoni, D., Cani, D. S., Cerini, M., Farina, D., Gavazzi, E., Maroldi, R., Adamo, M., Ammirati, E., Sinagra, G., Lombardi, C. M. and Metra, M. (2020) Cardiac involvement in a patient with coronavirus disease 2019 (COVID-19). JAMA Cardiology 5, 819-824. 
Jing, Z., Zhu, H., Yan, X., Chai, W. and Zhang, S. (2020) Recommendations from the Peking Union Medical College Hospital for the management of acute myocardial infarction during the COVID-19 outbreak. European Heart Journal 41, 1791-1794.

Li, B., Yang, J., Zhao, F., Zhi, L., Wang, X., Liu, L., Bi, Z. and Zhao, Y. (2020) Prevalence and impact of cardiovascular metabolic diseases on COVID-19 in China. Clinical Research in Cardiology 109, 531-538.

Murthy, S., Gomersall, C. D. and Fowler, R. A. (2020) Care for critically Ill patients with COVID-19. Journal of the American Medical Association 323, 1499-1500.

Panchal, A. R., Berg, K. M., Hirsch, K. G., Kudenchuk, P. J., Del Rios, M., Cabañas, J. G., Link, M. S., Kurz, M. C., Chan, P. S., Morley, P. T., Hazinski, M. F. and Donnino, M. W. (2019) 2019 American Heart Association focused update on advanced cardiovascular life support: use of advanced airways, vasopressors, and extracorporeal cardiopulmonary resuscitation during cardiac arrest: an update to the American Heart Association guidelines for cardiopulmonary resuscitation and emergency cardiovascular care. Circulation 140, e881-e894.

Pedersen, S. F. and Ho, Y. (2020) SARS-CoV-2: a storm is raging. Journal of Clinical Investigation 130, 2202-2205.

Rodríguez-Leor, O., Cid-Álvarez, B., Ojeda, S., Martín-Moreiras, J., Ramón Rumoroso, J., López-Palop, R., Serrador, A., Cequier, Á., Romaguera, R., Cruz, I., de Prado, A. P. and Moreno, R. (2020) Impacto de la pandemia de COVID-19 sobre la actividad asistencial en cardiología intervencionista en España. REC: Interventional Cardiology 2, 82-89. (In Spanish)

Roffi, M., Guagliumi, G. and Ibanez, B. (2020) The obstacle course of reperfusion for STEMI in the COVID-19 pandemics. Circulation 141, 1951-1953.

Roffi, M., Patrono, C., Collet, J., Mueller, C., Valgimigli, M., Andreotti, F., Bax, J. J., Borger, M. A., Brotons, C., Chew, D. P., Gencer, B., Hasenfuss, G., Kjeldsen, K., Lancellotti, P., Landmesser, U., Mehilli, J., Mukherjee, D., Storey, R. F. and Windecker, S. (2016) 2015 ESC guidelines for the management of acute coronary syndromes in patients presenting without persistent ST-segment elevation. European Heart Journal 37, 267-315.

Ruan, Q., Yang, K., Wang, W., Jiang, L. and Song, J. (2020) Clinical predictors of mortality due to COVID-19 based on an analysis of data of
150 patients from Wuhan, China. Intensive Care Medicine 46, 846-848. Shi, S., Qin, M., Shen, B., Cai, Y., Liu, T., Yang, F., Gong, W., Liu, X., Liang, J., Zhao, Q., Huang, H., Yang, B. and Huang, C. (2020) Association of cardiac injury with mortality in hospitalized patients with COVID-19 in Wuhan, China. JAMA Cardiology 5, 802-810.

Tavazzi, G., Pellegrini, C., Maurelli, M., Belliato, M., Sciutti, F., Bottazzi, A., Sepe, P. A., Resasco, T., Camporotondo, R., Bruno, R., Baldanti, F., Paolucci, S., Pelenghi, S., Iotti, G. A., Mojoli, F. and Arbustini, E. (2020) Myocardial localization of coronavirus in COVID-19 cardiogenic shock. European Journal of Heart Failure 22, 911-915.

The European Society for Cardiology.(2020) ESC guidance for the diagnosis and management of $\mathrm{CV}$ disease during the COVID-19 pandemic. Available at: https://www.escardio.org/Education /COVID-19-and-Cardiology/ESCCOVID-19-Guidance (Last update: 10 June 2020)

The World Health Organization. (2020) Director-General's remarks at the media briefing on 2019-nCoV on 11 February 2020. Available at: https://www.who.int/director-general/speeches/detail /who-director-general-s-remarks-at-the-media-briefing-o n-2019-ncov-on-11-february-2020 (Accessed: July 15, 2020).

Thiele, H., Ohman, E. M., de Waha-Thiele, S., Zeymer, U. and Desch, S. (2019) Management of cardiogenic shock complicating myocardial infarction: an update 2019. European Heart Journal 40, 2671-2683.

Thygesen, K., Alpert, J. S., Jaffe, A. S., Chaitman, B. R., Bax, J. J., Morrow, D. A. and White, H. D. (2018) Fourth universal definition of myocardial infarction (2018). Journal of the American College of Cardiology 72, 2231-2264.

Welt, F. G. P., Shah, P. B., Aronow, H. D., Bortnick, A. E., Henry, T. D., Sherwood, M. W., Young, M. N., Davidson, L. J., Kadavath, S., Mahmud, E. and Kirtane, A. J. (2020) Catheterization laboratory considerations during the coronavirus (COVID-19) pandemic. Journal of the American College of Cardiology 75, 2372-2375.

Zheng, Y., Ma, Y., Zhang, J. and Xie, X. (2020) COVID-19 and the cardiovascular system. Nature Reviews Cardiology 17, 259-260.

Zhou, F., Yu, T., Du, R., Fan, G., Liu, Y., Liu, Z., Xiang, J., Wang, Y., Song, B., Gu, X., Guan, L., Wei, Y., Li, H., Wu, X., Xu, J., Tu, S., Zhang, Y., Chen, H. and Cao, B. (2020) Clinical course and risk factors for mortality of adult inpatients with COVID-19 in Wuhan, China: a retrospective cohort study. The Lancet 395, 1054-1062. 